African Crop Science Journal by African Crop Science Society is licensed under a Creative Commons Attribution 3.0 Uganda License. Based on a work at www.ajol.info/ and www.bioline.org.br/cs DOI: http://dx.doi.org/10.4314/acsj.v26i1.2

\title{
ANALYSE DE LA VARIABILITE DES RENDEMENTS DU RIZ SELON LES VARIETES ET LES PRATIQUES CULTURALES : CAS DES PERIMETRES IRRIGUES DE TOULA, BONFEBA ET DE DIOMONA AU NIGER
}

\author{
I.Y. GAYA, I. MOSSI MAÏGA ${ }^{1}$, A. IDI $^{2}$ et A. HAOUGUI ${ }^{1}$ \\ Office National des Aménagements Hydro-agricoles (ONAHA), BP : 10697, Niamey, Niger \\ ${ }^{1}$ Institut National de la Recherche Agronomique du Niger (INRAN), BP : 429, Niamey, Niger \\ ${ }^{2}$ Université de Niamey, Faculté d'Agronomie, BP : 10960, Niamey, Niger \\ Auteur correspondant: fariagaya@yahoo.fr
}

(Received 18 September, 2017; accepted 15 February, 2018)

RÉSUMÉ

\begin{abstract}
La riziculture (Oryza sativa L.) est une activité très importante au Niger pour sa contribution à l'amélioration de la sécurité alimentaire et des revenus des populations. Cependant, malgré l'existence de plusieurs potentialités (potentiel irrigable, création de plusieurs périmètres irrigués), les nigériens continuent à être dépendants du riz en provenance de l'extérieur. Parmi les difficultés que rencontre la production rizicole, la principale reste la stagnation des rendements. C'est pour comprendre les causes qui sont à la base de cette stagnation que cette étude a été menée. L'objectif principal visé est de déterminer les facteurs induisant les écarts de rendements entre les variétés de riz selon les pratiques culturales des producteurs. L'étude s'est déroulée dans la région de Tillabéri, sur trois aménagements hydro-agricoles avec un échantillon de 50 producteurs repartis dans 5 villages. Pour atteindre cet objectif, l'étude s'est basée sur une phase d'enquête basée sur un questionnaire intégré dans un Smartphone, et une autre phase d'observation, basée sur la mise en place des placettes et sous-placettes dans les champs des producteurs afin de recueillir les informations sur les pratiques culturales du riz et d'évaluer les rendements obtenus. Les résultats obtenus ont montré que la gestion de la culture de riz est variable non seulement d'un périmètre à un autre, mais aussi d'un producteur à un autre, avec des conséquences sur le rendement. Des réductions importantes des écarts de rendement de 53 à $22 \%$ ont été obtenues en fonction de la variété, de la dose d'herbicide appliquée et le non-respect des âges des plants au repiquage.
\end{abstract}

Mots Clés : Oryza sativa, écarts de rendement, pratiques culturales, Tillabéri

\begin{abstract}
Rice (Oryza sativa L.) is very important in Niger for its contribution to improve food security and people's incomes. Despite the existence of several potentialities (irrigable potential, creation of several irrigated perimeters); Niger is still dependent on imports to meet its rice consumption requirements. Unfortunately, yields have stagnated. The objective of this study was to determine the factors that induce yield differentials between rice varieties based on the producers' cultural practices. The study was carried out in the Tillabéri region of Niger, on three irrigated perimeters, with a sample of 50 producers in 5 villages. The study was based on a survey with a questionnaire integrated into a Smartphone, and observation based on the demarcation of plots and subplots in the producers fields. Results obtained showed that the management of the crop was variable and spatially not only from one perimeter from one producer to another. Significant reductions in yield differentials of 53 to $22 \%$ were obtained based on the variety and applied herbicide dose.
\end{abstract}

Key Words: Oryza sativa, stagnation of yield, cultural practices, Tillaberi 


\section{INTRODUCTION}

Au Niger, on estime à 80000 ha la superficie mise en valeur chaque année pour la production du riz (Oryza sativa L.) avec environ 14 202,89 ha avec maitrise totale de l'eau encadrés par l' Office Nationale des Aménagements Hydro Agricoles (ONAHA) (ONAHA, 2013). La production nationale rizicole est estimée à 132030 tonnes de riz paddy dont environ 65860 tonnes produites sur les périmètres irrigués de la vallée du fleuve Niger (Sido et al., 2015) et 62030 tonnes hors aménagements (Issoufou et Issa, 2006).Cette production n'arrive à couvrir que le tiers des besoins croissants en riz de la population Nigérienne (Faivre Dupaigre et al., 2006). Le déficit est comblé par des importations estimées par l'Institut National des Statistiques (INS) à 330274 tonnes de riz blanc en 2013 pour une valeur d'environ 83470543456 FCFA (INS, 2014).

Pour renverser cette tendance, il est primordial d'améliorer la production nationale à travers l'augmentation des rendements qui stagnent aujourd'hui autour de $5 \mathrm{t} \mathrm{ha}^{-1}$ (ONAHA, 2013). Ces rendements peuvent être améliorés au vu des différents atouts dont dispose le pays comme en terme de surfaces irrigables et de sols favorables relevés par le Ministère de l'Hydraulique et de l'Environnement (MH/E) le document portant sur le Schéma directeur de mise en valeur et de gestion des ressources en eau du Niger dans un (MH/E, 1999). Par ailleurs, l'existence d'institutions de recherche et d'encadrement des producteurs, l'expérience des riziculteurs et l'homologation de plusieurs variétés de riz à haut rendements tel que spécifié par le Ministère de l'Agriculture et de l'Elevage (MAG/EL) constituent également des atouts non pour la riziculture nigérienne (MAG/EL, 2012) permettrait une réduction substantielle des écarts de rendements.

La différence entre le rendement potentiel et le rendement réel obtenu par les paysans est appelée écart de rendement (FAO, 2004).
Selon Evans et Fischer (1999) in Saito et al. (2017), «le rendement potentiel est défini comme le rendement maximal qui peut être obtenu à partir d'une culture dans un environnement donné, tel que déterminé par des modèles de simulation de cultures avec des hypothèses physiologiques et agronomiques plausibles ». Pour le cas de la riziculture, ce rendement potentiel dépend du système de riziculture. En effet, selon Fisher (2015) et Saito et al. (2017), "dans des conditions irriguées, le rendement potentiel est déterminé par le climat (rayonnement et température solaires), les caractéristiques variétales et les méthodes d'établissement des cultures, y compris la date de semis et la densité. Dans les conditions pluviales, le rendement potentiel est également affecté par la disponibilité de l'eau et se réfère au rendement limité à l'eau ». Plusieurs types de contraintes sont à la base des écarts de rendement. Il s'agit notamment des contraintes biophysiques, techniques, socio-économiques, institutionnelles, technologiques, de gestion des compétences (Dixon et al., 2001; Saito et al., 2017).

La consultation d'experts sur les écarts de rendements et la baisse de la productivité du riz, organisée par la FAO en 2000 (FAO, 2001), a constaté, selon les écologies, les régions et les saisons, des écarts substantiels entre les rendements potentiels et ceux obtenus par les riziculteurs pouvant aller de $10 \%$ à $60 \%$.Une étude sur l'identification des causes des écarts de rendements de riz de la variété IR841 au Togo, a montré des écarts de rendement de $40 \%$ à $60 \%$ selon l'écologie et de la gestion culturale de la variété (Komlan, 2011).

Au Niger, aucune étude n'a été menée dans l'évaluation des écarts de rendements de riz. Ainsi, l'objectif principal de cette étude est de déterminer les facteurs induisant les écarts de rendements entre les variétés de riz utilisées par les producteurs dans les périmètres irrigués. Il s'agit spécifiquement de recueillir les informations sur les pratiques culturales en irrigué et, de déterminer les causes des écarts 
de rendement par variété selon les pratiques culturales paysannes.

\section{MATERIELS ET METHODE}

Description du site. La présente étude a été menée dans le département de Tillaberi dont la pluviométrie moyenne annuelle est de $350 \mathrm{~mm}$ avec cependant de très fortes irrégularités interannuelles dans le temps et dans l'espace. Les températures moyennes annuelles varient de 25 à $45^{\circ} \mathrm{C}$ (Oumarou, 2006). L'hydrographie est marquée par la présence du fleuve dans la zone agricole et qui a favorisé la création de 18 périmètres irrigués rizicoles sur les 32 dont dispose la région (ONAHA, 2013).

Collected des donnees. La collecte des données a été faite en deux phases auprès d'un échantillon de 50 producteurs qui habitent les villages de Toula, Bonféba, Katanga, Beïnam et Korbolé, situés dans les communes rurales de Dessa et de Sakoira.

La première phase de collecte des données est basée sur l'administration d'un questionnaire intégré dans un Smartphone. La seconde phase est consacrée à la mise en place des placettes et sous-placettes dans les champs des producteurs afin de recueillir les informations sur les pratiques culturales et d'évaluer les rendements et les écarts de rendement. La situation des trois périmètres est indiquée dans la Figure 1 ci-dessous.

Les caractéristiques de ces périmètres sont consignées dans le Tableau 1 ci-dessous. Dans le champ de chaque producteur une placette de $200 \mathrm{~m}^{2}$ a été mise en place avec en son sein trois sous-placettes de $12 \mathrm{~m}^{2}$ chacune (voir Fig. 2). Les sous-placettes sont étiquetées $A$, $\mathrm{B}$ et C. Elles sont matérialisées par des piquets (>1 m) portant des étiquettes afin de facilité leur identification. Toutes les données ont été mesurées à l'intérieure des placettes et sous placettes.

La situation des trois périmètres est indiquée dans la Figure 2 ci-dessous. Les variables mesurées sont la superficie des parcelles, la densité du repiquage, le taux d'infestation par les adventices, le taux d'humidité des grains à la récolte, les rendements. Pour l'évaluation des rendements, trois placettes de $12 \mathrm{~m}^{2}$ ont été placées au hasard dans la parcelle du producteur.

Pour l'évaluation du rendement, le poids moyen des grains des trois sous placettes ont été pesé. A partir de ce poids moyen (P/12), le rendement moyen $\left(\mathrm{g} \mathrm{m}^{-2}\right)$ par sous placette ont été calculés en divisant le poids moyen par la superficie du sous placette $\left(12 \mathrm{~m}^{2}\right)$. Ensuite, il a été procédé à une extrapolation pour avoir les rendements en kilogrammes par hectare. La moyenne des pesées des trois placettes a été calculée pour avoir le rendement moyen. La teneur en humidité a été mesurée à l'aide

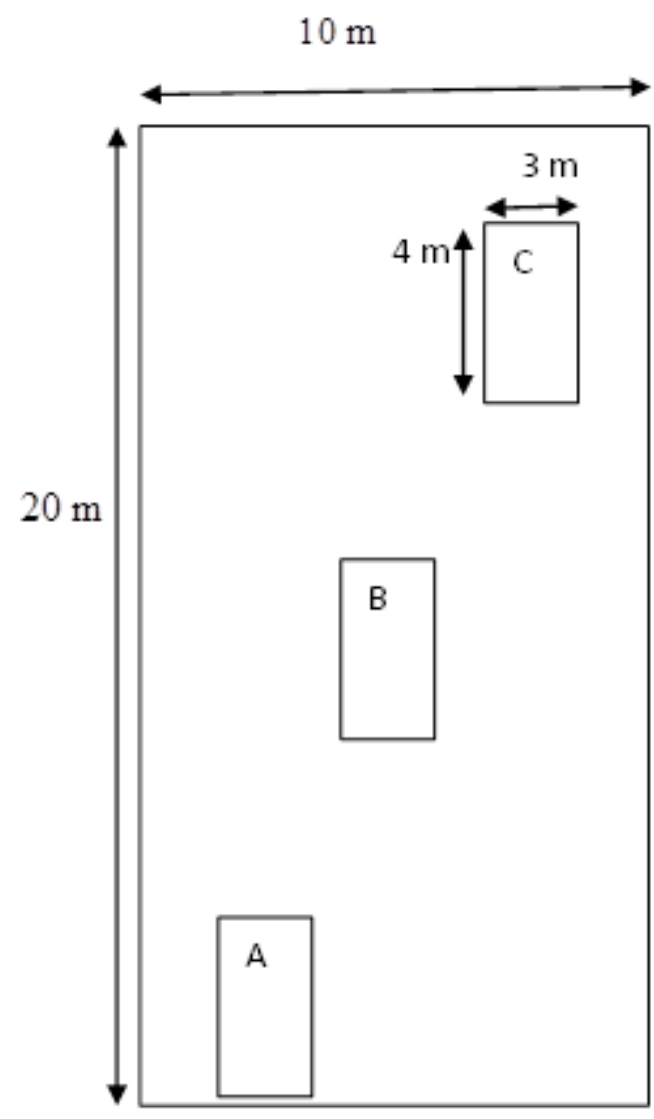

Figure 1. Schéma du dispositif installé dans les champs des producteurs. 
TABLEAU 1. Caractéristiques des périmètres irrigués étudiés

\begin{tabular}{lccccccc}
\hline $\begin{array}{l}\text { Nom du } \\
\text { périmètre }\end{array}$ & $\begin{array}{c}\text { Date de } \\
\text { création }\end{array}$ & $\begin{array}{c}\text { Taille } \\
\text { (ha) }\end{array}$ & $\begin{array}{c}\text { Nombre } \\
\text { GMP }\end{array}$ & $\begin{array}{c}\text { Nombre } \\
\text { villages }\end{array}$ & $\begin{array}{c}\text { Nombre } \\
\text { exploitants }\end{array}$ & $\begin{array}{c}\text { Encadrement } \\
\text { technique }\end{array}$ & $\begin{array}{c}\text { Organisation } \\
\text { de la } \\
\text { coopérative }\end{array}$ \\
\hline Bonféba & 1992 & 323,3 & 5 & 8 & 687 & Moyen & Moyenne \\
Diomona & 1992 & 409 & 8 & 12 & 716 & Moyen & Moyenne \\
Toula & 1975 & 243,2 & 6 & 7 & 669 & Moyen & Moyenne \\
\hline
\end{tabular}

GMP : Groupement Mutualiste des Producteurs. Source : INRAN (1996) et ONAHA (2010)

d'un humidimètre. Le rendement a été calculé selon la formule suivante :

Rendement $\left(\mathrm{kg} \mathrm{ha}^{-1}\right)=(10000 \times \mathrm{P} / 12) \times(100-$ $\mathrm{H}) /(100-14 \%)$, avec :

$\mathrm{P} / 12$ = poids moyen en $\mathrm{g}$ du paddy récolté dans $12 \mathrm{~m}^{2} ; \mathrm{H}=$ Teneur en eau des grains

Enfin, l'écart de rendement, en pourcentage, a été calculé selon la formule de la FAO (2004): Ecart de rendement $(\%)=$ Rendement potentiel ( $\left.\mathrm{kg} \mathrm{ha}{ }^{-1}\right)$ - Rendement moyen obtenu/ Rendement potentiel $\left(\mathrm{kg} \mathrm{ha}^{-1}\right)$ x 100

Ainsi donc :

(i) Les rendements moyens par variété selon l'âge des plants au repiquage ont été déterminés pour calculer les écarts de rendement.

(ii) Les rendements moyens par variété selon la densité de repiquage ont été déterminés pour calculer les écarts de rendement.

(iii) Les rendements moyens par variété selon la dose d'herbicide appliquée ont été déterminés pour calculer les écarts de rendement.

(iv) Les rendements moyens par variété selon le nombre de désherbage manuel effectué ont été déterminés pour calculer les écarts de rendement.

(v) Les rendements moyens par variété selon la dose d'engrais minéral appliquée ont été déterminés pour calculer les écarts de rendement.
Les informations sur les pratiques de gestion des cultures ont été collectées à travers des entretiens avec les producteurs.

Analyse des données. Les données ont été saisies avec le tableur Microsoft Excel qui a aussi servi à la construction des graphiques et aux calculs des moyennes. L'analyse de variance (ANOVA) a été exécutée à l'aide du logiciel statistique GenStat Discovrery (Wim et al., 2004) qui a permis de faire également de comparaison par le test de comparaison des moyennes de Student-Newman-Keuls au seuil de $5 \%$.

\section{RESULTATS}

Les variétés de riz produites sur les périmètres Quatre variétés de riz ont été rencontrées dont deux homologuées (Gambiaka et l'IR15) et deux variétés locales (Somba et Kardjikoyo). Selon les techniciens des coopératives qui exploitent ces périmètres, les variétés $S o m b a$ et Kardjikoyo sont issues d'une sélection massale à partir de la variété IR15.C'est pourquoi il a été considéré dans cette étude que ces variétés aient le même rendement potentiel que la variété IR15. Les principales caractéristiques des différentes variétés sont indiquées dans le Tableau 2.

L'enquête menée auprès des 50 producteurs a révélé que la variété de riz la plus cultivée a été la Somba avec un taux d'utilisation de $60 \%$ principalement sur le périmètre de Bonféba. Elle a été suivie de la Gambiaka et l'IR15 qui ont occupé 


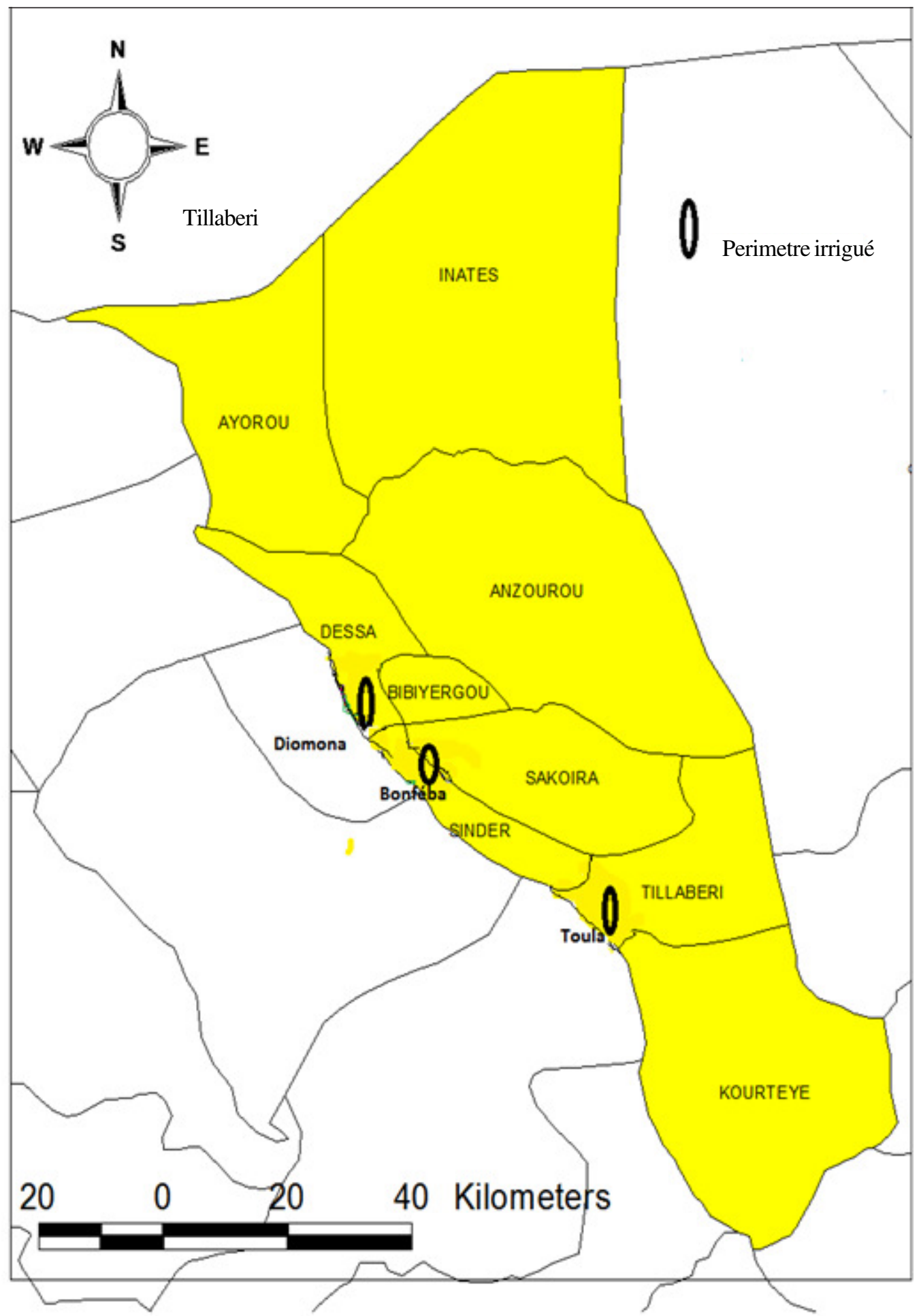

Figure 2. Situation des 3 périmètres irrigués dans le département de Tillaberi. 
respectivement la deuxième $(24 \%)$ et la troisième place $(10 \%)$ en termes d'utilisation par les producteurs. La variété Kardjikoyo était la moins cultivée des variétés. Des quatre variétés recensées, Gambiaka, l'IR15 et la Kardjikoyo ont été cultivées sur le périmètre de Bonféba, la Gambiaka et l'IR15 sur le périmètre de Toula alors que la Somba seule sur le périmètre de Diomona (Tableau 3).

Le repiquage dans les trois périmètres irrigués. Le repiquage était la méthode de mise en place de la culture du riz au niveau des trois périmètres. Les plantules issues des pépinières ont été repiquées en ligne. Les riziculteurs se distinguent le plus souvent par l'âge des plantules au repiquage et par le nombre de plants repiqués par poquet. Le Tableau 4 présente l'âge moyen des plantules au repiquage selon le périmètre.

Il ressort de l'analyse du Tableau 4 que l'âge moyen des plantules au repiquage a varié légèrement entre les trois périmètres. C'est à Diomona qu'on a observé les plus vieux plants (43 jours) au repiquage. Les résultats de l'analyse de variance relative à l'âge des plantules au repiquage ont montré qu'il existe une différence significative entre les producteurs en ce qui concerne l'âge des plants au repiquage $(\mathrm{P}=0,019)$.

La densité de repiquage est relative au nombre de poquets par $\mathrm{m}^{2}$. Le Tableau 5 présente la densité moyenne des poquets par $\mathrm{m}^{2}$ et aussi et par hectare selon le périmètre.

La plus grande densité de repiquage (220 000 poquets ha ${ }^{-1}$ ) est observée sur le périmètre de Toula. Les périmètres de Diomona et de Bonféba ont occupé la deuxième et la troisième position avec respectivement 190000 et 180 000 poquets $\mathrm{ha}^{-1}$. Le Tableau d'analyse de variance relative au nombre du poquet $\mathrm{ha}^{-1}$ montre qu'il existe une différence hautement significative $(\mathrm{P}=0.008)$ au sein des riziculteurs sur les trois périmètres irrigués.

La gestion des adventices sur les périmètres irrigués. Cinq grandes familles d'adventices ont été collectées au cours de 
TABLEAU 3. Taux d'utilisation des 4 variétés utilisées selon le périmètre

\begin{tabular}{lcccc}
\hline Périmètres & \multicolumn{4}{c}{ Taux d'utilisation (\%) des 4 variétés utilisées } \\
\cline { 2 - 5 } & Gambiaka & IR1529 & Somba & Kardjikoyo \\
\hline Bonféba & 4 & 10 & 0 & 6 \\
Diomona & 0 & 0 & 60 & 0 \\
Toula & 20 & 0 & 0 & 0 \\
Total & 24 & 10 & 60 & 6 \\
\hline
\end{tabular}

TABLEAU 4. Ages moyens des plantules au repiquage

\begin{tabular}{lccc}
\hline $\begin{array}{l}\text { Ages des plantules au repiquage } \\
\text { (en jours après semis) }\end{array}$ & Bonféba & Diomona & Toula \\
\hline Minimum & 25 & 25 & 25 \\
Moyenne & 43 & 36 & 33 \\
Maximum & 60 & 67 & 45 \\
\hline
\end{tabular}

TABLEAU 5. Densité moyenne de repiquage

\begin{tabular}{lcc}
\hline Périmètre & Moyenne nombre de poquet $\mathrm{m}^{-2}$ & Moyenne nombre de poquet/ha \\
\hline Toula & 22 & 220000 \\
Bonféba & 18 & 180000 \\
Diomona & 19 & 190000 \\
\hline
\end{tabular}

cette étude. Il s’agit des Cypéracées, des Malvacées, des Graminées, des Sphenocleacées et des Onagracées. Le taux de couverture des adventices dans les parcelles était globalement très faible, voire nul. Le Tableau 6 présente la nature des cinq principaux adventices les plus rencontrées par ordre d'importance.

Les espèces les plus rencontrées sur les périmètres irrigués sont Portula oleracea et Marsilia minuta. Elles ont été secondées par Cyperus difformis et Oryza longistaminata dont le taux de couverture a été variable d'un périmètre à un autre.
La gestion des adventices par les riziculteurs sur les trois périmètres s'est faite soit par voie chimique, soit de façon manuelle. Désherbage chimique.

Le désherbage chimique intervient le plus souvent 2 semaines après le repiquage. Il est pratiqué par l'ensemble des producteurs enquêtés. L'herbicide qui est utilisé par les producteurs des 3 périmètres est le Londax dont la matière active est Bensulfuron méthyle. Cependant, bien que les périodes d'application soit identiques chez tous les producteurs, il a existé une différence fondamentale par rapport aux doses utilisées (Tableau7) 
On a constaté que seuls les producteurs du périmètre de Bonféba ont utilisé la dose de Londax recommandée $\left(80 \mathrm{~g} \mathrm{ha}^{-1}\right)$. A Diomona et à Toula, les doses appliquées ont été inférieures à la norme recommandées.

Désherbage manuel. Il intervient le plus souvent avant l'application de l'engrais minéral, mais le nombre de désherbage varie d'un producteur à un autre (Tableau 8).

L'analyse des variances a montré qu'il n'existe pas de différence significative $(\mathrm{P}=$ 0,61 ) au niveau des trois périmètres par rapport au nombre de désherbage. Il ressort que les producteurs font en moyenne 2 désherbages manuels à Diomona et à Bonféba et 3 à Toula.

Fertilisation dans les trois périmètres irrigués. Deux types d'engrais sont utilisés par les producteurs sur les 3 périmètres : l'engrais organique et les engrais chimiques. L'engrais organique appliqué est le fumier tandis que le NPK et l'urée ont été les deux types d'engrais minéraux qui sont utilisés (Fig. 3)

Le pourcentage d'utilisation du fumier est faible, moins de $40 \%$ des riziculteurs. Mais ce taux a varié légèrement d'un périmètre à un autre. Il est de $40 \%$ pour le périmètre de Toula contre $36 \%$ à Diomona et $30 \%$ à Bonféba. Il faut aussi noter qu'il a existé une autre forme d'engrais organique utilisé dans une moindre mesure par l'ensemble des riziculteurs. Il s'agit des résidus des cultures et des adventices qui sont incorporés dans le sol pendant le labour. Par contre, $100 \%$ des producteurs sur les trois périmètres utilisent l'engrais minéral. Cependant, il a été constaté que sur les

TABLEAU 6. Classement des principales espèces d'adventices répertoriées

\begin{tabular}{|c|c|c|c|c|}
\hline \multirow[t]{2}{*}{ Familles } & \multirow[t]{2}{*}{ Nom des espèces } & \multicolumn{3}{|c|}{ Rang des espèces/ périmètre } \\
\hline & & Bonféba & Diomona & Toula \\
\hline Cypéracées & Cyperusdifformis & & & 4 \\
\hline Onagracées & HeterantheracallifoliaRchb & 5 & & \\
\hline Sphenocleacée & SphenocleazeylanicaGaertner & & 5 & \\
\hline \multirow[t]{3}{*}{ Graminées } & Echinochloasp & & & 5 \\
\hline & Echinochloacolona & 3 & 3 & \\
\hline & Oryzalongistaminata & 4 & 4 & 3 \\
\hline \multirow[t]{2}{*}{ Malvacées } & Marsila minuta & 2 & 2 & 2 \\
\hline & Portulaoleracea & 1 & 1 & 1 \\
\hline
\end{tabular}

TABLEAU 7. Dose moyenne d'herbicide utilisé par les producteurs sur les trois périmètres

\begin{tabular}{lc}
\hline Périmètre & $\begin{array}{c}\text { Dose moyenne d'herbicide } \\
\text { utilisée }\left(\mathrm{g} \mathrm{ha}^{-1}\right)\end{array}$ \\
\hline Bonféba & 80 \\
Diomona & 48 \\
Toula & 46 \\
\hline
\end{tabular}

TABLEAU 8. Nombre moyen de désherbage effectué par périmètre

\begin{tabular}{lc}
\hline Périmètre & $\begin{array}{c}\text { Moyenne nombre } \\
\text { de désherbage }\end{array}$ \\
\hline Bonféba & 2 \\
Diomona & 2 \\
Toula & 3
\end{tabular}


périmètres, les producteurs se différencient dans la pratique de l'application de l'engrais minéral. D'abord sur le type de l'engrais minéral utilisé, ensuite par la quantité appliquée et enfin par le nombre de fractionnement (Tableau 9).

C'est le périmètre de Bonféba qui a enregistré la dose moyenne la plus importante avec $578 \mathrm{~kg} \mathrm{ha}^{-1} \mathrm{~d}^{\prime}$ engrais minéral utilisé en 2 fractions avec un maximum avoisinant les 800 $\mathrm{kg} \mathrm{ha}^{-1}$ et un minimum d'environ $400 \mathrm{~kg} \mathrm{ha}^{-1}$.
Cette dose est sensiblement égale à celle appliquée sur le périmètre de Toula $(532 \mathrm{~kg}$ ha $^{-1}$ ) apportée en 3 fractionnements. Le périmètre de Diomona a eu la plus faible dose, $340 \mathrm{~kg} \mathrm{ha}^{-1}$ et la dose maximale la plus importante, $1712 \mathrm{~kg} \mathrm{ha}^{-1}$.

Le Tableau 10 ci-dessous présente l' analyse de variance des pratiques culturales au niveau des 3 périmètres irrigués.

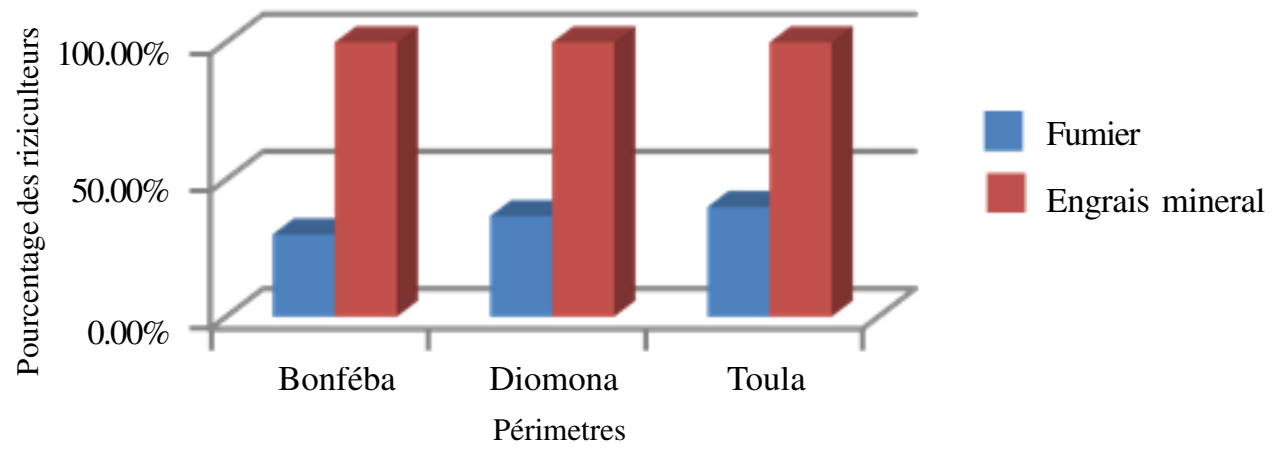

Figure 3. Pourcentage des riziculteurs selon le type de l'engrais utilisé au niveau des trois périmètres.

TABLEAU 9. Dose moyenne d'engrais minéral appliqué sur les 3 périmètres et le nombre d'application

\begin{tabular}{lcccc}
\hline Périmètre & $\begin{array}{c}\text { Dose moyenne } \\
\text { d'engrais minéral } \\
\left(\mathrm{kg} \mathrm{ha}^{-1}\right)\end{array}$ & $\begin{array}{c}\text { Dose maximum } \\
\left(\mathrm{kg} \mathrm{ha}^{-1}\right)\end{array}$ & $\begin{array}{c}\text { Dose minimum } \\
\left(\mathrm{kg} \mathrm{ha}^{-1}\right)\end{array}$ & $\begin{array}{c}\text { Nombre moyen } \\
\text { d'application }\end{array}$ \\
\hline Bonféba & 578 & 782 & 393 & 2 \\
Diomona & 340 & 1712 & 102 & 2 \\
Toula & 532 & 830 & 382 & 3 \\
\hline
\end{tabular}

TABLEAU 10. Analyse de variance des pratiques culturales au niveau des 3 périmètres irrigués

\begin{tabular}{lrrrrr}
\hline Source de variation & d.f. & s.s. & m.s. & v.r. & F pr. \\
\hline Densité de repiquage & 2 & 550444. & 275222. & 5.35 & 0.008 \\
Age des plants au repiquage & 2 & 5138077. & 2569038. & 4.32 & 0,019 \\
Nombre d'application de l'engrais & 2 & 39769. & 19885. & 2.96 & 0.061 \\
Nombre de désherbage effectué & 2 & 1.3333 & 0.6667 & 1.88 & 0.614 \\
dose herbicide & 2 & 5.249 & 2.625 & 1.25 & 0.297 \\
Dose d'engrais minéral & 2 & 8.528 & 4.264 & 0.11 & 0.893 \\
\hline
\end{tabular}


Variabilité des écarts de rendement par variété selon les pratiques culturales

Rendement moyen par variété selon l'âge des plantules repiquées. La Figure 4 présente les écarts de rendement selon l'âge des plants au repiquage.

De l'analyse de la Figure 4, on a observé les trois types d'âge des plants au repiquage chez deux variétés à savoir IR15 et Somba, tandis que chez les deux autres variétés (Gambiaka et Kardjikoyo) on n'a observé que deux type d'âge des plants au repiquage. Ainsi chez les variétés IR15 et Gambiaka, les écarts de rendement n'ont pas différé significativement selon que les plants sont repiqués à l'âge de 25 jours ou plus de 50 jours. Il en est de même chez la variété Somba. Par contre au niveau de la variété Kardjikoyo bien que l'écart ne soit pas significatif entre les plants repiqués de 25-30 jours et ceux repiqués à l'âge de 30-45 jours, l'écart de rendement obtenu au niveau de cette variété est très faible par rapport aux écarts de rendement observés au niveau des trois variétés de riz.
Ecart de rendement selon la densité de repiquage. La Figure 5 présente les écarts de rendement selon la densité de repiquage. Au niveau de la variété IR15, on observe que l'écart de rendement qui est réduit de $35 \%$ avec les faibles densités $\left(<150000\right.$ poquets ha $\left.^{-1}\right)$ est passé autour de $40 \%$ avec des plants repiqués avec des densités moyennes (entre 150000 et 250000 poquets ha ${ }^{-1}$ ) et fortes (> 250000 poquets ha ${ }^{-1}$ ).

Par contre au niveau de la variété Somba, la différence entre les écarts de rendements n'est pas significative $(P=0,540)$ entre les densités moyennes et fortes, l'écart de rendement par rapport au rendement potentiel est très important (39-40\%).

Enfin pour les 2 autres variétés (Gambiaka et Kardjikoyo), la comparaison est impossible car on observe la même densité culturale partout où ces 2 variétés sont cultivées. Cependant, l'écart de rendement obtenu au niveau de la variété Kardjikoyo est réduit jusqu' à $29 \%$ avec des densités moyennes.

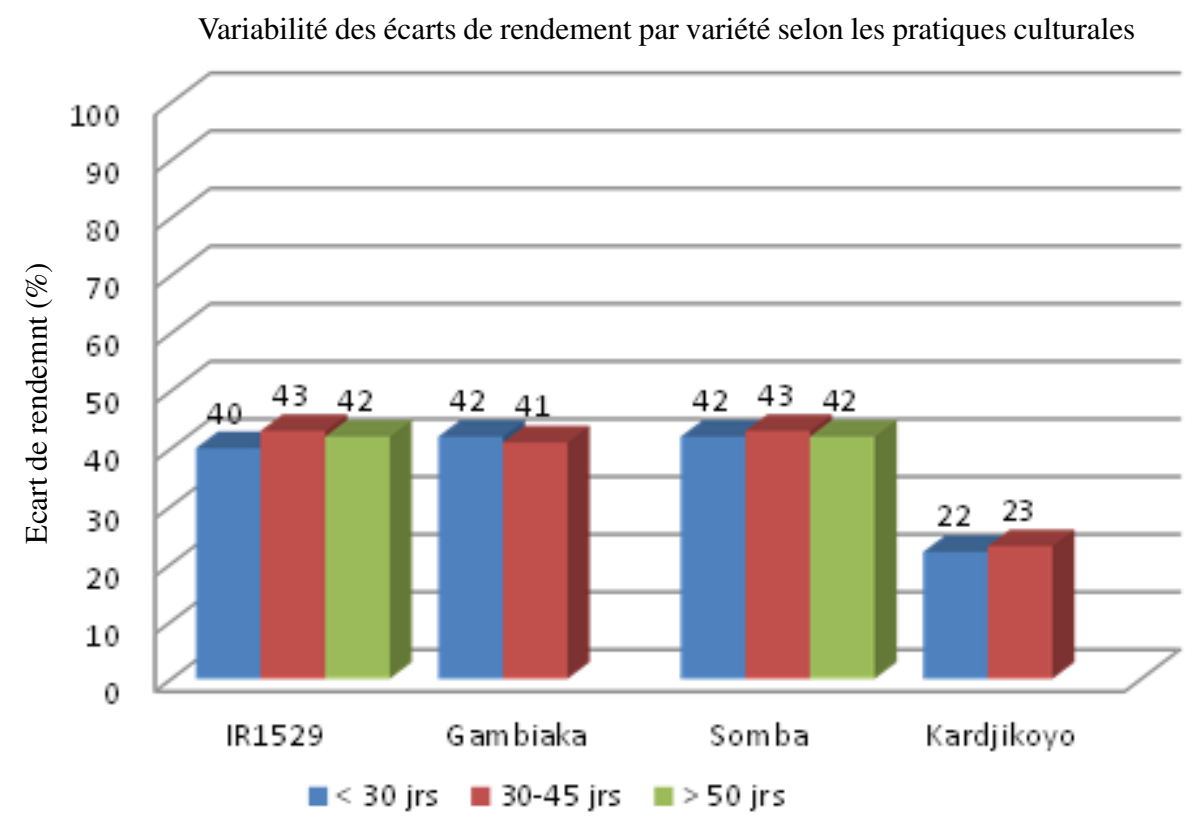

Age moyen des plants (jours)repiqués et les varietés de riz

Figure 4. Ecart de rendement du riz par variété et selon l'âge des plantules au repiquage. 
Ecart de rendement par variété selon la Figure 7 présente les écarts de rendement selon dose de l'herbicide appliquée. La Figure 6 présente les écarts de rendement selon la dose de l'herbicide appliqué.

A la lecture de la Figure 6, on a constaté que les réductions des écarts de rendements ont augmenté avec la quantité de l'herbicide appliquée pour toutes les variétés à l'exception de la variété Kardjikoyo.

le nombre de désherbage manuel effectué.

De l'analyse de la Figure 7, on a observé que les pourcentages d'écart de rendements ont diminué dans la majorité des cas avec le nombre de désherbage effectué pour toutes les variétés.

Rendement moyen par variété selon la dose de l'engrais minéral appliqué. La Figure 8

Ecart de rendement par variété selon le présente les écarts de rendement selon la dose nombre de désherbage manuel effectué. La d'engrais minéral appliqué.

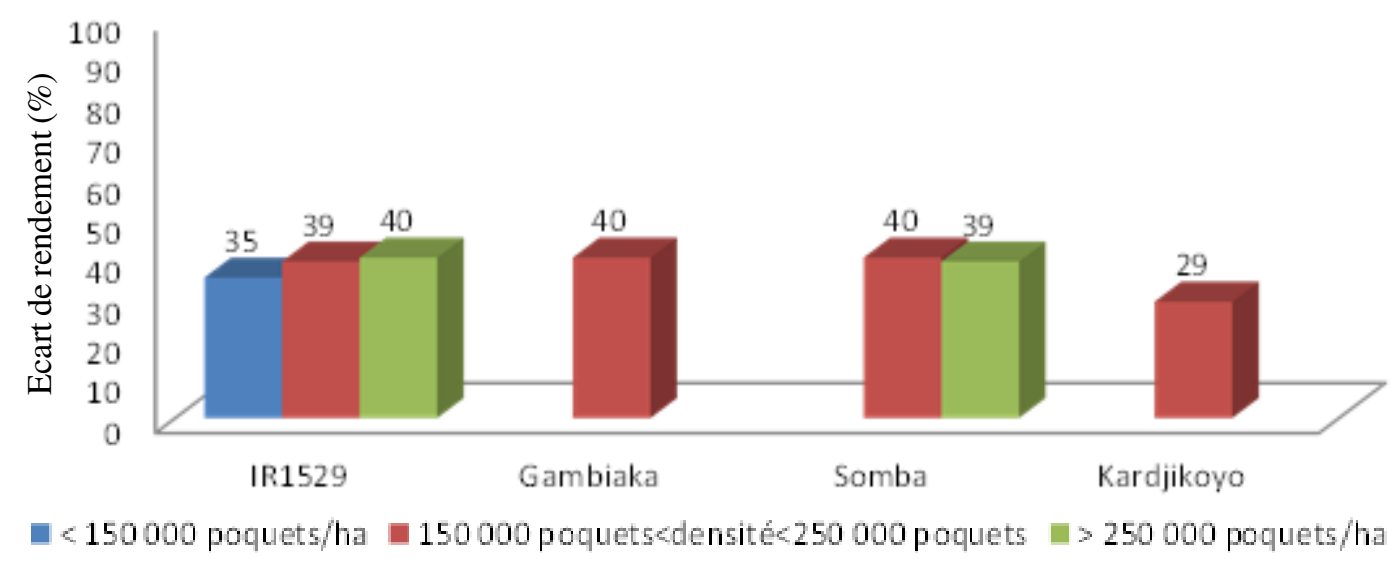

Densité de repiquage (nombre de poquets/ha) et les varietés de riz

Figure 5. Ecart de rendement du riz par variété et selon la densité de repiquage.

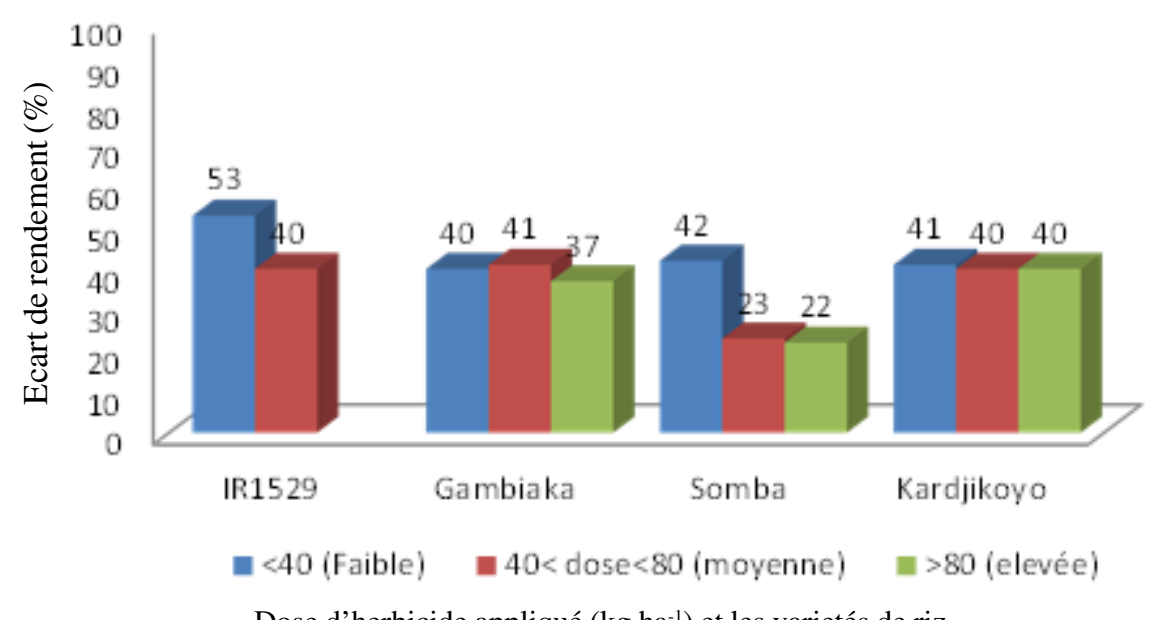

Figure 6. Ecart de rendement du riz par variété et selon la dose d'herbicide appliquée. 
De l'analyse de la Figure 8, il a été ressorti que c'est seulement au niveau de la variété Kardjikoyo qu'on a observé une différence significative entre les différentes doses d'engrais appliquées. Ainsi, l'écart de rendement a été réduit de 42 à $22 \%$ avec une dose d'engrais supérieure à $450 \mathrm{~kg} \mathrm{ha}^{-1}$. Par contre chez les trois autres variétés on a observé que les producteurs ont appliqué, dans leur ensemble, plus de $300 \mathrm{~kg} \mathrm{ha}^{-1}$ d'engrais minéral et l'écart de rendement a tourné autour de $40 \%$.
Le Tableau 11 ci-dessous présente l'analyse de variance de la variabilité des rendements selon les 4 variétés et pratiques culturales

\section{DISCUSSION}

Les résultats de la présente étude ont montré des différences dans la gestion de la culture du riz non seulement entre les périmètres, mais aussi entre les riziculteurs ce qui, n'est pas sans conséquence sur les différents rendements obtenus.

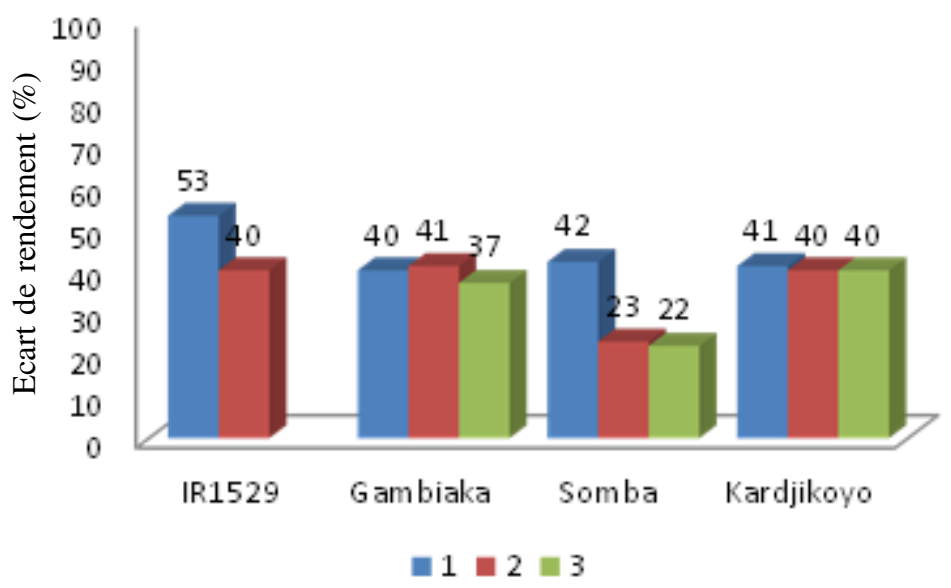

Nombre de desherbage effectué et les varietés de riz

Figure 7. Ecart de rendement par varieté et selon le nombre de désherbage effectué.

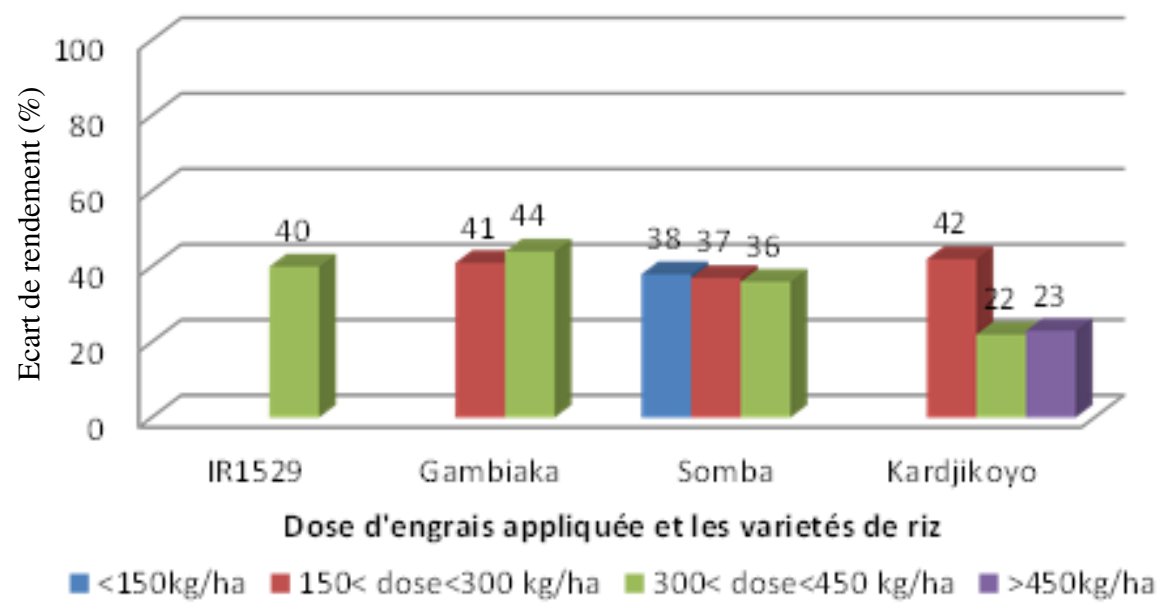

Figure 8. Ecart de rendement par variété et selon la dose de l'engrais minéral appliqué. 
TABLEAU 11. Analyse de variance de la variabilité des rendements selon les 4 variétés et pratiques culturales

\begin{tabular}{|c|c|c|c|c|c|c|}
\hline Variétés & Source de variation & d.f & s.s & m.s & v.r & F pr \\
\hline Gambiaka & dose engrais minéral & 8 & 87056 & 10882 & 5.15 & 0.329 \\
\hline Gambiaka & densité de repiquage & 8 & 6.8 & 8.5 & 4.25 & 0,359 \\
\hline Gambiaka & dose herbicide appliquée & 8 & 8473.2 & 941.5 & 2.3 & 0.038 \\
\hline Gambiaka & Nombre de désherbage effectué & 8 & 8.4 & 1.05 & 2.1 & 0.49 \\
\hline Gambiaka & Age des plants & 8 & 520.9 & 65.11 & 1.3 & 0.594 \\
\hline IR15 & Age des plants & 6 & 2.1371 & 3.5619 & & \\
\hline IR15 & densité de repiquage & 6 & 1880.538 & 208.949 & 47.62 & $<0.01$ \\
\hline IR15 & dose herbicide appliquée & 6 & 87056 & 10882 & 5.15 & 0.0329 \\
\hline IR15 & dose engrais minéral & 6 & 11666.9 & 1944.5 & & \\
\hline IR15 & Nombre de désherbage effectué & 6 & 4.85714 & 0.80952 & & \\
\hline kardjikoyo & Age des plants & 2 & 16.6667 & 8.3333 & & \\
\hline kardjikoyo & densité de repiquage & 2 & 2.3267 & 1.1633 & & \\
\hline kardjikoyo & dose herbicide appliquée & 2 & 1112.67 & 556.33 & & \\
\hline kardjikoyo & dose engrais minéral & 2 & 48553 & 24276 & & \\
\hline kardjikoyo & Nombre désherbage effectué & 2 & 2 & 1 & & \\
\hline Somba & Nombre de désherbage effectué & 15 & 14.5333 & 0.9689 & 2.81 & 0.03 \\
\hline Somba & dose engrais minéral & 15 & 571246 & 38083 & 1.79 & 0.141 \\
\hline Somba & densité de repiquage & 15 & 2.591 & 1.727 & 0.95 & 0.540 \\
\hline Somba & Age des plants & 15 & 778.63 & 51.91 & 0.87 & 0.608 \\
\hline Somba & dose herbicide appliquée & 15 & 38685.17 & 3516.83 & 11.38 & 0.0001 \\
\hline
\end{tabular}

Tout d'abord, il a été observé des différences dans l'utilisation des variétés. En effet, il y a une prédominance de la variété locale «Somba » avec un taux d'utilisation de $60 \%$ sur l'ensemble des trois périmètres. Outre le choix des variétés, l'étude a montré de grandes différences dans la pratique de la gestion du riz au niveau des trois périmètres tel que la densité du repiquage, l'âge moyen des plants repiqués et la gestion des engrais organiques et chimiques.

Ainsi la différence de la densité de repiquage observée d'une part entre les producteurs et les faibles densités de repiquage (inférieure à 250000 poquets $\mathrm{ha}^{-1}$ ) entre les périmètres d'autre part, pourraient être expliquée par l'utilisation ou non des cordes comme équipement conçu pour respecter la densité de repiquage $(20 \mathrm{~cm} \times 20 \mathrm{~cm})$. En dehors de la différence dans les densités de repiquage, il faut ajouter également les variabilités dans l'âge des plants repiqués.

Cela s'explique par la concurrence des temps des travaux entre la culture du riz dans les périmètres irrigués et celle des autres cultures (mil, sorgho, niébé) dans les champs communément appelés champs dunaires pour signifier qu'il s'agit de champs à sol à prédominance sableux. En effet, le chevauchement des travaux est le principal facteur qui impacte les activités du repiquage en saison d'hivernage car cette période est surtout caractérisée par la rareté de la main d'œuvre (Ekoye et al., 1996).

Par ailleurs, l'étude a montré qu'avec la variété Kardjikoyo, l'écart de rendement est de $29 \%$, alors qu'il tourne autour de $40 \%$ chez les autres variétés. La réduction de l'écart de 
rendement (29\%) observé au niveau de la variété Kardjikoyo peut être expliquée par plusieurs raisons. Tout d'abord, Kardjikoyo est exclusivement cultivée sur le périmètre de Bonféba où les résultats ont montré des doses les plus élevées d'engrais minéral $(578 \mathrm{~kg}$ $\mathrm{ha}^{-1}$ ) et d'herbicide. Aussi, l'évaluation des dégâts de maladies ont montré que c'est le périmètre le moins infesté.

L'étude a également démontré qu'au sein de la même variété il existe des variations des écarts de rendement selon les pratiques culturales adoptées par les producteurs.

Ainsi les résultats ont montré qu'au niveau de toutes les variétés, les écarts de rendement diffèrent significativement selon la dose d'herbicide appliquée. D'après ces résultats, il est possible d'augmenter le rendement du riz et donc de réduire les écarts de rendement (5 à 15\% selon les variétés) par l'application des doses d'herbicide supérieures à $80 \mathrm{~g} \mathrm{ha}^{-1}$ qui est la dose recommandée par l'encadrement. L'inefficacité de la dose recommandée s'explique d'une part par la mauvaise qualité du produit, mais aussi par la mauvaise application (moment d'application) et l'utilisation du même produit sur les périmètres depuis plusieurs années. Selon Arraudeau (1998) certains herbicides sont inefficaces quand ils sont utilisés sur le même terrain plusieurs années de suite, induisant l'apparition des phénomènes de résistance. Cela explique pourquoi, malgré l'utilisation des herbicides, les producteurs procèdent encore à des désherbages manuels dont le nombre varie de un à trois. La différence entre les écarts de rendement observée proviendrait certainement de l'effectivité des désherbages notamment le premier, au moment opportun, cela est confirmé par le faible écart de rendement observé selon la quantité d'herbicide appliqué.

Cependant, malgré la série de désherbages effectués par les producteurs, certaines espèces d'adventices comme Portula oleracea et Marsilia minuta sont les plus répertoriées. Le faible taux de couverture de ces adventices serait lié à l'application de l'herbicide Londax. L'herbicide est appliqué 2 semaines après le repiquage et avant le désherbage manuel au moment où les adventices sont en pré-germination. L'autre raison pourrait être le statut hydrique des parcelles où la présence quasi permanente de l'eau pourrait gêner les adventices par asphyxie. Pour ce qui est de la densité du repiquage, au niveau de la variété IR15, on a observé un écart de rendement de $35 \%$ avec les faibles densités $\left(<150000\right.$ poquets $\left.\mathrm{ha}^{-1}\right)$. Il passe autour de $40 \%$ avec des plants repiqués avec des densités moyennes (entre 150000 et 250000 poquets ha ${ }^{-1}$ ) et fortes (> 250000 poquets ha $^{-1}$ ).Par contre au niveau de la variété $S o m b a$, la différence entre les écarts de rendements n'est pas significative entre les densités moyennes et fortes. Les importants écarts de rendements (39-40\%) observés peuvent être liés aux potentiels de tallage des variétés.

Pour les variétés Somba et Kardjikoyo, la comparaison est impossible car on observe la même densité culturale partout où elles sont cultivées. Cependant, l'écart de rendement obtenu au niveau de la variété Kardjikoyo est de $29 \%$ avec des densités moyennes.

Par ailleurs bien qu'il n'existe pas de différence significative entre les producteurs pour les autres variables (âges des plants au repiquage et dose et d'engrais), il a été observé des écarts selon la variété utilisée. Ainsi chez les variétés IR15 et Gambiaka, les écarts de rendement ne diffèrent pas significativement selon que les plants sont repiqués à l'âge de 25 jours ou plus de 50 jours. Il en est de même chez la variété Somba. Par contre au niveau de la variété Kardjikoyo, bien que l'écart ne soit pas significatif entre les plants repiqués à 25- 30 jours et ceux repiqués à l'âge de 30-45 jours, l'écart de rendement obtenu au niveau de cette variété est très faible par rapport aux écarts de rendement observés au niveau des trois autres variétés. La première situation s'expliquerait par le fait que les plants sont repiqués au moment opportun. En effet, selon 
les recommandations inscrites dans le Catalogue National des Espèces et Variétés Végétales (MAG/EL, 2017) l'âge optimum des plants au repiquage est de 25 à 30 jours pendant la campagne humide $(\mathrm{SH})$. La réduction importante de l'écart de rendement observé chez la variété Kardjikoyo justifie son utilisation par les riziculteurs bien qu'elle ne soit pas recommandée sur les périmètres irrigués car non homologuée. Aussi, certains producteurs ont affirmé qu'en plus de son rendement élevé, cette variété a l'avantage d'avoir un cycle court (120 jours) par rapport aux autres variétés.

Pour ce qui est de l'engrais minéral, les résultats ont montré que malgré le surdosage, les écarts de rendement tournent autour de $40 \%$ pour toutes les variétés. Cela pourrait s'expliquer par une mauvaise application et les périodes optimales d'application. Pour les producteurs, qui prétendent que leurs terres sont pauvres, des apports de grandes quantités d'engrais permettraient d'avoir les bons rendements. Les producteurs avancent en effet que leurs terres sont fatiguées et de ce fait exigent beaucoup d'engrais. La baisse de la fertilité des sols a été confirmée par l'étude réalisée par Attikou (1999) sur les périmètres rizicoles de Tillabéri, et qui a montré que la fertilité des sols des périmètres irrigués est non seulement faible mais aussi et qu'elle a baissé de manière spectaculaire après 5 à 6 ans de mise en culture. On doit en effet noter que les engrais sont indispensables à l'intensification de la riziculture. Or, comme le dit Basso (2011) dans sa thèse, l'application de fortes doses d'urée et le non respect des périodes d'application peuvent s'avérer nocive à la riziculture. Les fortes doses favorisent, en effet, un développement excessif du feuillage qui accroit les risques de dégâts causés par les maladies notamment le flétrissement bactérien (Basso, 2011). Il est donc nécessaire que les doses à appliquer respectent les normes mais aussi et surtout qu'au niveau de la recherche les normes soient de plus en plus actualisées en tenant compte de l'évolution de la fertilité des sols.
$\mathrm{Si}$, un accent particulier doit être accordé à l'apport du fumier dans la riziculture, dans notre étude, il a été remarqué un faible taux d'utilisation du fumier par moins de $50 \%$ des producteurs et qu'ils justifient par les difficultés à le transporter sur les périmètres. En effet, l'éloignement des parcelles par rapports aux habitats, et parfois le manque de moyen de transport adéquat (charrette, transport sur la tête par les enfants) constituent les handicaps et expliquent la faible utilisation du fumier. Les producteurs n'apportent pas aussi le fumier car ils estiment qu'il est source de beaucoup de maladies.

Par ailleurs, une étude similaire conduite par Kamlan au Togo en 2011 avec la variété IR841, a montré un écart de rendement de $40 \%$ à $60 \%$ selon l'écologie et de la gestion culturale de la variété.

De façon générale, ces écarts de rendements sont nettement inférieurs à ceux observés sur certaines spéculations au Niger. En effet, selon le document de l'Initiative 3N, sur les 30 dernières années, le rendement moyen le plus élevé du mil a été de $529 \mathrm{~kg}$ ha${ }^{1}$ obtenu en 2010, ce qui est de deux à trois fois (soit un écart de 50 à 66\%) en deçà du potentiel de productivité enregistré en station de recherche. Par ailleurs, les écarts de rendement (qui varient de 22 à 53\%) obtenu au cours de cette étude cadrent parfaitement avec ceux obtenus par la FAO (2004). En effet, selon la FAO les écarts de rendement du riz dans le monde varient de 10 à $60 \%$. En se référant à ces données, on peut comprendre que les écarts de rendements au Niger sont légèrement proches des écarts de rendements mondiaux du riz.

\section{CONCLUSION}

Les résultats de la présente étude ont montré que les quatre variétés cultivées n'expriment pas leurs rendements potentiels. Les écarts de rendement obtenus varient de 22 à $53 \%$. Ces écarts sont liés aux pratiques culturales telles que la densité de repiquage, la dose d'herbicide 
appliquée, la période de désherbage et surtout le choix de la variété cultivée.

De ces résultats, on peut conclure que les principales causes des écarts de rendement sont les pratiques culturales et la variété cultivée.

Par ailleurs, le non-respect des paquets technologiques est parfois lié à certains facteurs socioéconomiques qui n'ont pas été prises en compte dans la présente étude. En effet, si les producteurs font du surdosage ou sous dosage des engrais ou des herbicides, c'est parce qu'ils n'ont pas suffisamment de connaissance sur le mode d'emploi de ces intrants. Ainsi, le respect de la dose recommandée n'est pas forcément suivi d'efficacité si la période d'application n'est pas respectée. Cela pose le problème du nonrespect du calendrier cultural qui s'explique d'une part par la mauvaise gestion des périmètres, mais aussi par la rareté de la main d'œuvre.

De tous ces constats, on note que les aspects socio économiques sont les principales causes des écarts de rendements. Il est donc souhaitable que cette étude puisse continuer en prenant en compte le facteur pédologique et socio-économique.

Les résultats de la présente étude n'ont pas montré de différence significative pour les pratiques culturales telles que l'âge des plants au repiquage et la dose d'engrais minéral apporté au sein d'une même variété. Les analyses doivent être poussées en prenant en compte simultanément tous les facteurs, en faisant recours aux analyses en composante principales (ACP), afin de voir sur quels facteurs on doit agir pour réduire considérablement les écarts de rendements.

\section{REMERCIEMENT}

Nous exprimons nos remerciements aux personnes suivantes qui nous ont aidés à développer ce travail. Les Directeurs des périmètres de Toula, Bonféba et Diomona respectivement. Mr. AMADOU Boureïma,
Mr. IDRISSA Oumarou et Mr. MOUSSA Koukou, qui nous ont facilité le contact avec les producteurs dans leurs périmètres.

\section{REFERENCES BIBLIOGRAPHIQUES}

Arraudeau, M. 1998. Le riz irrigué. Tome $1 \mathrm{Et}$ Tome II.Paris, Maisonneuve et Larose. 229pp.

Attikou, A. 1999. Suivi de la fertilité des sols des aménagements hydro-agricoles : Cas des périmètres rizicoles de Bonféba et Lata. Niamey, Office National des Aménagements Hydro-agricoles. 39pp.

Basso, A. 2011. Biologie de Xanthomnas oryzae pv. oryzae (Ishiyama) responsible du flétrissement bactérien du riz (Oryza sativa L.) au Niger. Thèse de l'Université Abdou Moumouni de Niamey. Niamey, Université de Niamey, Niger. 148pp.

Ekoye, A., Tankari, D. et Schalbroek, J. 1996. Rapport de l'année 1995 Office National des Aménagements Hydroagricoles. Niamey, Niger. Ministère du Développement Rural, de l'Hydraulique et de l'Environnement. 30pp.

Faivre Dupaigre, B., Allahoury, A., Eychenne, D., Gado, B. et Koure, A. 2006. Propositions pour une politique rizicole au Niger, Niamey, Institut de Recherches et des d'Applications des Méthodes de Développement. 193pp.

Food and Agriculture Organization (FAO). 2001. Écarts de rendements et baisse de productivité de la production de riz. Actes de la Consultation d'experts sur l'écart de rendements et la baisse de productivité de la production de riz. FAO. Rome, Italy. $2 \mathrm{pp}$.

Food and Agriculture Organization (FAO). 2004. Riz et limitation de l'écart de Rendement. FAO. Rome, Italy. 2pp.

Institut National des Statistiques .2014. Bulletin Trimestriel du Commerce Extérieur. Edition Mars 2014. Niamey, Niger. INS.17pp. 
Issoufou, A. et Issa, G. 2006. Détermination des surfaces et estimation de la production de riz hors aménagements hydro-agricole. Rapport d'étude. Niamey, Programme d'Appui à la Filière Riz. 43pp.

Komlan, A.A. 2011. Identification des causes des écarts de rendements dans deux zones de production de riz au Togo. 18pp.

Ministère de l'Agriculture et de l'Elevage. 2017. Annuaire National 2017 sur la disponibilité en semences améliorées au Niger. Niamey, Niger. MAG/EL. 124pp.

Ministère de l'Agriculture. 2012. Catalogue National des Espèces et Variétés Végétales (CNEV), le riz. Edition 2012. Niamey, MAG. 39pp.

Ministère de l'Hydraulique et de l'Environnement. 1999. Schéma directeur de mise en valeur et de gestion des ressources en eau du Niger : le référentiel du secteur de l'eau et de l'assainissement au Niger. Niamey, Ministère de l'hydraulique et de l'environnement. 151pp. Office National des Aménagements Hydro agricoles (ONAHA). 2013. Rapport annuel 2012. Niamey, Niger. 46pp.

Office National des Aménagements Hydro agricoles (ONAHA). 2010. Rapport annuel de la direction de mise en valeur. Office National des Aménagements Hydro agricoles du Niger. Niamey, Niger. 47pp.
Oumarou, O. 2006. Suivi des activités sur le périmètre irrigué de Daïbéri, rapport de stage. Faculté d'Agronomie de l'Université Abdou Moumouni . Niamey, Niger. 27pp. Sido, A., Saminou, E., Hassane, A., Mossi Maïga, I., Maï Alimi, O., Yacouba, I., Bouzou, A., Hassane, M., Baye I. et Seyni, I. 2015. Etat des lieux de la riziculture au Niger. Niamey, Ministère de l'agriculture/ PPAAO-WAAPP-WAAPP. 92pp.

Saito, K., van Oort. P., Dieng, I., Johnson, J.M., Niang, A., Ahouanton, K., Amakoe, D.A., Tanaka, A., Senthilkumar, K., Vandamme, E., Akakpo, C., Segda, Z., Bassoro, I., Mapiemfu, D., Allarangaye, M.D., Gbakatchetche, H., Bayuh, B.A., Jaiteh, F., Bam, R.K., Dogbe, W., Sékou K., Rabeson, R., Kamissoko, K., Maïga Mossi, I., Bakare, O.S., Mabone, F.L., Gasore, E.R., Baggie, I., Kajiru, G.J., Ablede, K.A. et Nanfumba, D. 2007. Yield gap analysis towards meeting future rice demand. In: Sasaki, T. (Ed.). Achieving sustainable cultivation of rice: Cultivation, pest and disease management. Burleigh Dodds Science Publishing, Cambridge, UK 2:157-182.

Wim, B., Roger, S. et Ric, C. 2004. GenStat Discovery Edition for everyday use. ICRAF Nairobi, Kenya. 114 pp. 